\title{
ON A CLASS OF INTEGRAL EQUATIONS
}

JOANNE ELLIOTT

1. Introduction. We shall discuss the integral equation

(1) $g(x)=P \cdot \frac{1}{\pi} \int_{-a}^{+a} \frac{f(t)}{t-x} d t+\lambda \int_{-a}^{+a} h(x, t) f(t) d t,-a<x<+a$,

where $g$ and $h$ are given and $f$ is unknown. The number $a$ may be finite or infinite. The first integral is taken in the sense of a Cauchy principal value. For $h(x, t) \equiv 0$, this reduces to a well known integral equation of airfoil theory and has been treated extensively, mainly by Fourier methods, cf. [2]. E. Reissner [3] has applied this method to (1) in the case where $h(x, t)$ is an odd polynomial in $x-t$ and $a$ is finite. He showed that a formal expansion of the assumed solution may be obtained in this case by solving finitely many linear equations. We may note that if $\lambda=1$ and $a=1$ and

$$
h(x, t)=\frac{1}{2} \cot \pi\left(\frac{x-t}{2}\right)-\frac{\pi^{-1}}{x-t},
$$

we obtain another well known equation.

It would seem of interest to consider the more general case. ${ }^{1}$ The use of Fourier expansions suggests Hilbert space methods, and it is natural to modify the equation formally by introducing a suitable weight function $w(x)$. That is, instead of equation (1), we consider the equivalent equation

$$
g(x)=P \cdot \frac{1}{\pi} \int_{-a}^{+a} \frac{f(t) w(t)}{t-x} d t+\lambda \int_{-a}^{+a} h(x, t) f(t) w(t) d t .
$$

Of all the choices of weight functions the most suitable turns out to be the one which makes

$$
T f=P \cdot \frac{1}{\pi} \int_{-a}^{+a} \frac{f(t) w(t)}{t-x} d t
$$

an isometric mapping from one Hilbert space onto another. If $a=\infty$, then $w(t) \equiv 1$ has this property. In the finite interval if we let $a=1$

Presented to the Society, December 28, 1951; received by the editors June 14, 1951.

1 The author has recently found that Šerman [5] has considered a similar problem, but has used a different approach. He does not use Hilbert space, and his conditions are of a local character. 
for simplicity, it will be shown that this is achieved by choosing

$$
w(x)=(1-x)^{1 / 2}(1+x)^{-1 / 2}{ }^{2}
$$

The transformation $T^{-1}$ will reduce (2) to a Fredholm equation. Two systems of Jacobi polynomials provide the necessary tools.

$\S \S 2$ and 3 will be devoted to the case $a<\infty$ and the fourth to the case $a=\infty$.

2. The Hilbert spaces for $a<\infty$. We desire to find a weight function $w(x)$ such that (3) will transform a set of polynomials orthonormal with respect to $w(x)$ into a set of polynomials orthonormal with respect to another weight function $w_{1}(x)$. It is easily seen that (3) will transform a polynomial of degree $n$ into another polynomial of degree $n$ if and only if

$$
P \cdot \frac{1}{\pi} \int_{-1}^{+1} \frac{w(t)}{t-x} d t=\text { const. } \neq 0 .
$$

Recall that we have put $a=1$ here for simplicity. All admissible solutions of this equation are given by

$$
w(t)=c_{1}(1-t)^{1 / 2}(1+t)^{-1 / 2}+c_{2}\left(1-t^{2}\right)^{-1 / 2}
$$

where $c_{1}$ and $c_{2}$ are constants. We shall choose

$$
w(t)=(1-t)^{1 / 2}(1+t)^{-1 / 2} .
$$

This will lead to

$$
w_{1}(t)=(1+t)^{1 / 2}(1-t)^{-1 / 2}
$$

as Lemma 1 will show. It will be clear from symmetry that the rôles of (5) and (6) could be interchanged. A system of polynomials orthonormal with respect to the weight function (5) are the Jacobi polynomials $P_{n}^{(1 / 2,-1 / 2)}(x)$ which we shall denote by $P_{n}(x)$. The polynomials corresponding to (6) are the Jacobi polynomials $P_{n}^{(-1 / 2,1 / 2)}(x)$ and will be denoted by $Q_{n}(x)$. These polynomials are defined by

$$
\begin{aligned}
& P_{n}(\cos \theta)=\alpha_{n} \frac{\sin [(n+1 / 2) \theta]}{\sin \theta / 2}, \\
& Q_{n}(\cos \theta)=\alpha_{n} \frac{\cos [(n+1 / 2) \theta]}{\cos \theta / 2}
\end{aligned}
$$

where $\alpha_{n}$ is a norming constant.

We define

\footnotetext{
${ }^{2}$ For general $a$, use $w(x)=(a-x)^{1 / 2}(a+x)^{-1 / 2}$.
} 


$$
T_{1} g=-P \cdot \frac{1}{\pi} \int_{-1}^{+1} \frac{g(t) w_{1}(t)}{t-x} d t .
$$

The following two lemmas are contained in much more general results of Achieser [1]. In proving Lemma 2 we use Achieser's argument with the simplifications permitted by our special weight functions. His method for Lemma 1 is unnecessary in our case.

LEMMA 1. With the weight functions defined in (5) and (6), we have

$$
\begin{aligned}
T P_{n} & =-Q_{n}, \\
-T_{1} Q_{n} & =P_{n} .
\end{aligned}
$$

Proof. To prove (9), we have to show that

$$
P \cdot \frac{1}{\pi} \int_{0}^{\pi} \frac{\sin [(n+1 / 2) \theta] \sin \theta / 2}{\cos \theta-\cos \phi} d \theta=-\frac{1}{2} \frac{\cos [(n+1 / 2) \phi]}{\cos \phi / 2}
$$

We may rewrite the left side as

$$
P \cdot \frac{1}{2 \pi} \int_{0}^{\pi} \frac{\{\cos n \theta-\cos [(n+1) \theta]\}}{\cos \theta-\cos \phi} d \theta
$$

and use the well known formula

$$
\frac{1}{\pi} P \cdot \int_{0}^{\pi} \frac{\cos m \theta}{\cos \theta-\cos \phi} d \theta=\frac{\sin m \phi}{\sin \phi}, \quad 0<\phi<\pi,
$$

to obtain the result. We may prove (10) in a similar way.

LEMMA 2. The transformation (3) maps the Hilbert space $H[w]$ of functions which are square integrable with respect to $w(x)$ isometrically onto the Hilbert space $H\left[w^{-1}\right]$ of functions which are square integrable with respect to $1 / w(x)$. The inverse of $T$ is $T_{1}$.

Proof. Any element $f \in H[w]$ can be expressed as $f(x)$ $=\sum_{j=0}^{\infty} c_{j} P_{j}(x)$, the series converging in norm. We let $f_{n}(x)$ $=\sum_{j=0}^{n} c_{j} P_{j}(x)$ and $g_{n}(x)=-\sum_{j=0}^{n} c_{j} Q_{j}(x)$. From Lemma 1 , we know that $g_{n}(x)=T f_{n}(x)$. We know also that $g_{n}$ converges to an element $g \in H[w]$ as $n \rightarrow \infty$, that is, $g(x)=-\sum_{j=0}^{\infty} c_{j} Q_{j}(x)$. We cannot conclude, however, that $g=T f$ until we know that $T$ is a continuous transformation from $H[w]$ to $H\left[w_{1}\right]$. To show this, we first observe that

$$
f(x) w(x) \in L_{2 r /(r+1)}
$$

for any $1<r<2$, by Hölder's inequality. From a well known theorem of Marcel Riesz, cf. [5, p. 132], 


$$
\begin{aligned}
\int_{-\infty}^{+\infty}\left|g_{n}(x)-g_{m}(x)\right|^{2 r /(r+1)} d x & \\
& \leqq A_{r} \int_{-1}^{+1}\left|\left[f_{n}(x)-f_{m}(x)\right] w(x)\right|^{2 r /(r+1)} d x
\end{aligned}
$$

Hence $g_{n}$ converges to $T f$ in the mean of $2 r /(r+1)$. Since $g_{n}$ converges to $g$ in the norm of $H[w]$, it follows that $g=T f$ almost everywhere.

3. Reduction to a Fredholm equation. Suppose that

$$
\begin{aligned}
& \int_{-1}^{+1}|h(x, t)|^{2} w_{1}(x) d x<\infty, \quad-1<t<+1, \\
& \int_{-1}^{+1} \int_{-1}^{+1}|h(x, t)|^{2} w_{1}(x) w(t) d x d t<\infty, \\
& \int_{-1}^{+1}|g(x)|^{2} w_{1}(x) d x<\infty .
\end{aligned}
$$

In what follows all series expansions are to be considered as series converging in the norms of the corresponding Hilbert spaces. We can then expand $h$ and $g$ as follows:

$$
h(x, t)=\sum_{i, j} c_{i j} Q_{i}(x) P_{j}(t), g(x)=\sum_{i} g_{i} Q_{i}(x) .
$$

Suppose a solution $f(t) \in H[w]$ of equation (2) exists. Applying $T_{1}$ to both sides we have

$$
\tilde{g}(x)=f(x)+\lambda \int_{-1}^{+1} \tilde{h}(x, t) f(t) w(t) d t
$$

a.e.

where $\tilde{h}(x, t)=-\sum_{i, j} c_{i j} P_{i}(x) P_{j}(t)$ and $\tilde{g}=T_{1} g$. Conversely, we may apply $T$ to both sides of (16), to conclude that the solutions must satisfy equation (2). Therefore equation (16) is a Fredholm equation equivalent to (2). Furthermore, if $c_{i j}=c_{j i}$, then the new equation will have a symmetric kernel.

We see also that (16) or (2) is equivalent to the system of linear equations

$$
-g_{k}=f_{k}-\lambda \sum_{j} c_{k j} f_{j}, \quad k=0,1,2, \cdots .
$$

Condition (14) on $h(x, t)$ implies that $\sum_{i, j}\left|c_{i j}^{2}\right|<\infty$. Hence the linear transformation on $l^{(2)}$ with matrix $\left[c_{i j}\right]$ is completely continuous. It is well known (cf. [4]) that in this case, for each fixed $\lambda$, 
the study of (17) may be reduced to the study of a finite system of equations. A condition which assures a unique solution of (2) is $\sum_{i, j}\left|c_{i j}^{2}\right|<|\lambda|^{-1}$. If $h(x, t)$ is a polynomial in $x-t$ with real coeffcients $\alpha_{i}$, then this reduces to

$$
\sum_{i, j} \alpha_{i} \alpha_{j}\left(\begin{array}{c}
i+j \\
{[(i+j) / 2]}
\end{array}\right)\left(\begin{array}{c}
i+j+1 \\
{[(i+j+2) / 2]}
\end{array}\right) \frac{(-1)^{i+j} \pi^{2}}{2^{i+j}}<|\lambda|^{-1}
$$

where $[x]$ denotes the greatest integral part of $x$.

4. The infinite interval. In this case, we again reduce (1) to an equation of the Fredholm type. We shall assume that

$$
\int_{-\infty}^{+\infty}|h(x, t)|^{2} d x<\infty
$$

for all $t$ and is in $L[-\infty,+\infty]$.

Instead of the transformation (3), we shall use

$$
T f=P \cdot \frac{1}{\pi} \int_{-\infty}^{+\infty} \frac{f(t)}{t-x} d t
$$

the Hilbert transform (cf. [6, chap. V]). The inverse of $T$ in $L_{2}[-\infty,+\infty]$ is given by

$$
T_{1} g=-P \cdot \frac{1}{\pi} \int_{-\infty}^{+\infty} \frac{g(t)}{t-x} d t
$$

and if $g=T f$, then

$$
\int_{-\infty}^{+\infty}|g(t)|^{2} d t=\int_{-\infty}^{+\infty}|f(t)|^{2} d t
$$

We shall use the following lemma.

LeMma 2. Let $f(t) \in L_{2}[-\infty,+\infty]$. If $F(t)$ is the Fourier transform of $f$, then the Fourier transform of $T f$ is given by $-i F(x) \operatorname{sgn} x$.

A proof of this lemma may be found in [6, chap. V, Theorems 90 and 91 ].

We apply $T_{1}$ to both sides of (1). We first wish to show that

$$
\begin{aligned}
T_{1}\left\{\int_{-\infty}^{+\infty} h(x, t) f(t) d t\right\} & \\
= & -\int_{-\infty}^{+\infty} f(t)\left\{\text { P.V. } \frac{1}{\pi} \int_{-\infty}^{+\infty} \frac{h(u, t)}{u-x} d u\right\} d t .
\end{aligned}
$$


Note that condition (18) assures that the function in braces on the left side of (22) is in $L_{2}$.

Let

$$
h(x, t)=\underset{A \rightarrow \infty}{\lim .} \frac{1}{(2 \pi)^{1 / 2}} \int_{-A}^{+A} e^{-i x u} H(u, t) d u .
$$

Then, assuming for the moment that the following formal operations are justified, we have

$$
\begin{aligned}
\int_{-\infty}^{+\infty} f(t) h(x, t) d t & \\
& =\int_{-\infty}^{+\infty} f(t)\left\{\lim _{A \rightarrow \infty} \frac{1}{(2 \pi)^{1 / 2}} \int_{-A}^{+A} e^{-i x u} H(u, t) d u\right\} d t \\
& =\lim _{A \rightarrow \infty} \frac{1}{(2 \pi)^{1 / 2}} \int_{-A}^{+A} e^{-i x u}\left\{\int_{-\infty}^{+\infty} f(t) H(u, t) d t\right\} d u .
\end{aligned}
$$

The function in braces in the last integral is in $L_{2}$ by Parseval's formula. By Lemma 2 this gives

$$
\begin{aligned}
& T_{1}\left\{\int_{-\infty}^{+\infty} f(t) h(x, t) d t\right\} \\
& \quad=\lim _{A \rightarrow \infty} \frac{1}{(2 \pi)^{1 / 2}} \int_{-A}^{+A} e^{-i x u} i \operatorname{sgn} u\left\{\int_{-\infty}^{+\infty} f(t) H(u, t) d t\right\} d u \\
& \left.\quad=i \int_{-\infty}^{+\infty} f(t) \lim _{A \rightarrow \infty} \frac{1}{(2 \pi)^{1 / 2}} \int_{-A}^{+A} H(u, t) e^{-i x u} \operatorname{sgn} u d u\right\} d t,
\end{aligned}
$$

which is just (22).

We must now prove (24). We have

$$
\begin{aligned}
& \int_{-\infty}^{+\infty}\left|\int_{-\infty}^{+\infty} f(t)\left[h(x, t)-\frac{1}{(2 \pi)^{1 / 2}} \int_{-A}^{+A} e^{-i x u} H(u, t) d u\right] d t\right|^{2} d x \\
& \leqq\|f\|^{2} \int_{-\infty}^{+\infty}\left\{\int_{-\infty}^{+\infty}\left|h(x, t)-\frac{1}{(2 \pi)^{1 / 2}} \int_{-A}^{+A} e^{-i x u} H(u, t) d u\right|^{2} d t\right\} d x
\end{aligned}
$$

which is equal to

$$
\|f\|^{2} \int_{-\infty}^{+\infty}\left\{\int_{|u|>A}|H(u, t)|^{2} d u\right\} d t .
$$

For the proof of this last statement, see $[6$, p. 75]. That (27) goes 
to zero with $A$ follows from (18). This completes the proof of (24).

The equation then reduces to

$$
\tilde{g}(x)=f(x)+\lambda \int_{-\infty}^{+\infty} f(t) \tilde{h}(x, t) d t
$$

a.e.

where $\tilde{g}=T_{1} g$ and $\tilde{h}(x, t)=T_{1} h(x, t)$ where the last transformation is taken with respect to $x$. Furthermore $\tilde{h}(x, t)$ also satisfies (18). By a similar argument on (28) with $T$ it is clear that (28) is equivalent to (1) with $a=\infty$. The integral transform in (28) is completely continuous and the equation (28) will have a unique solution in $L_{2}$ provided

$$
\int_{-\infty}^{+\infty} \int_{-\infty}^{+\infty}|\tilde{h}(x, t)|^{2} d x d t<|\lambda|^{-1}
$$

5. Comparison with other methods. The usual treatment of (3) and Reissner's treatment of (1) is equivalent to using instead of (5) and (6) the weight functions

$$
w(x)=\left(1-x^{2}\right)^{-1 / 2} \text { and } w_{1}(x)=\left(1-x^{2}\right)^{1 / 2}
$$

respectively, and reducing to a system of linear equations by direct substitution into (2). We cannot use the mapping $T$ as we did before since with this weight function, $w(x)$, any constant is taken into zero by $T$. This transformation takes the Tchebycheff polynomials $T_{n}(x)$ of the first kind into the Tchebycheff polynomials $U_{n-1}(x)$ of the second kind. The system of equations obtained by this procedure is no longer of the Fredholm type (17).

\section{ReferenCes}

1. N. I. Achieser, On some inversion formulas for singular integrals (in Russian), Bull. Acad. Sci. URSS (Izvestia) Sér. Math. vol. 9 (1945).

2. G. Hamel, Integralgleichungen, Springer, 1949.

3. E. Reissner, Solution of a class of singular integral equations, Bull. Amer. Math. Soc. vol. 51 (1945).

4. F. Riesz, Les systèmes d'équations linéaires à une infinité d'inconnues, GauthierVillars, 1913.

5. D. I. Šerman, On a case of regularization of singular integral equations (in Russian), Akademiya Nauk SSSR. Prikladnaya Matematika i Mehanika vol. 15 (1951) pp. 75-82.

6. E. C. Titchmarsh, Introduction to the theory of the Fourier integral, Oxford, 1948.

\section{Mount Holyoke College and Swarthmore College}

